\title{
The design, implementation, and performance of the Astro-H SXS aperture assembly and blocking filters
}

\author{
Caroline A. Kilbourne ${ }^{* a}$, Joseph S. Adams ${ }^{\text {a.b }}$, Petar Arsenovic ${ }^{a}$, Travis Ayers ${ }^{\mathrm{c}}$, Meng P. Chiao ${ }^{\text {a.b }}$, \\ Michael J. DiPirro a, Megan E. Eckart ${ }^{\mathrm{a}}$, Ryuichi Fujimoto ${ }^{\mathrm{d}}$, John D. Kazeva ${ }^{\mathrm{a}, \mathrm{e}}$, \\ Richard L. Kelley ${ }^{\mathrm{a}}$, Kari L. Kripps ${ }^{\mathrm{f}}$, Bruce Lairson ${ }^{\mathrm{c}}$, Maurice A. Leutenegger ${ }^{\mathrm{a}, \mathrm{b}}$, Heidi Lopez ${ }^{\mathrm{c}}$, \\ Dan McCammon ${ }^{\mathrm{f}}$, Daniel S. McGuinness ${ }^{\mathrm{a}}$, Kazuhisa Mitsuda ${ }^{\mathrm{g}}$, Samuel J. Moseley ${ }^{\mathrm{a}, \mathrm{h}}$, \\ F. Scott Porter ${ }^{\mathrm{a}}$, Andrea N. Schweiss ${ }^{\mathrm{a}}$, Yoh Takei ${ }^{\mathrm{g}}$, Rosemary S. Thorpe ${ }^{\mathrm{a}, \mathrm{i}}$, \\ Tomomi Watanabe $^{\mathrm{a}, \mathrm{j}}$, Noriko Y. Yamasaki ${ }^{\mathrm{g}}$, Seiji Yoshida $^{\mathrm{k}}$ \\ ${ }^{a}$ NASA Goddard Space Flight Center, Greenbelt, MD 20771 USA, \\ ${ }^{b}$ University of Maryland, Baltimore County, Baltimore, MD 21250 USA, \\ ${ }^{\circ}$ Luxel Corporation, Friday Harbor, WA 98250 USA, \\ ${ }^{d}$ Kanazawa University, Kakuma-machi, Kanazawa, Ishikawa 920-1192, Japan, \\ ${ }^{\mathrm{e} S G T}$, Inc., Greenbelt, MD 20770 USA, \\ ${ }^{\mathrm{f}}$ Department of Physics, University of Wisconsin, Madison, WI 53706 USA, \\ ${ }^{g}$ ISAS/JAXA, 3-1-1 Yoshinodai, Chuo-ku, Sagamihara, Kanagawa 252-5210, Japan, \\ ${ }^{\mathrm{h}}$ ADNET Systems, Inc., Bethesda, MD 20817 USA, \\ ${ }^{\mathrm{i} B}$ Bastion Technologies Inc., Houston, TX 77058 USA, \\ ${ }^{j}$ University of Maryland, College Park, MD 20742, USA, \\ ${ }^{\mathrm{k}}$ Sumitomo Heavy Industries, Ltd., 5-2 Soubiraki-cho, Niihama, Ehime 792-8588, Japan
}

\begin{abstract}
The calorimeter array of the JAXA Astro-H (renamed Hitomi) Soft X-ray Spectrometer (SXS) was designed to provide unprecedented spectral resolution of spatially extended cosmic $\mathrm{x}$-ray sources and of all cosmic $\mathrm{X}$-ray sources in the Fe-K band around $6 \mathrm{keV}$, enabling essential plasma diagnostics. The properties that make the SXS array a powerful x-ray spectrometer also make it sensitive to photons from the entire electromagnetic band, and particles as well. If characterized as a bolometer, it would have a noise equivalent power (NEP) of $<4 \times 10^{-18} \mathrm{~W} /(\mathrm{Hz})^{0.5}$. Thus it was imperative to shield the detector from thermal radiation from the instrument and optical and UV photons from the sky. Additionally, it was necessary to shield the coldest stages of the instrument from the thermal radiation emanating from the warmer stages. Both of these needs are addressed by a series of five thin-film radiation-blocking filters, anchored to the nested temperature stages, that block long-wavelength radiation while minimizing $\mathrm{x}$-ray attenuation. The aperture assembly is a system of barriers, baffles, filter carriers, and filter mounts that supports the filters and inhibits their potential contamination. The three outer filters also have been equipped with thermometers and heaters for decontamination. We present the requirements, design, implementation, and performance of the SXS aperture assembly and blocking filters.
\end{abstract}

Keywords: x-ray calorimeter, microcalorimeter, radiation-blocking filters, aperture cylinder, Astro-H, Hitomi, SXS

\section{INTRODUCTION}

The detectors ${ }^{1}$ of the Hitomi SXS instrument ${ }^{2}$ are anchored to a $50 \mathrm{mK}$ heat sink provided by a multi-stage and partially redundant cooling system ${ }^{3,4}$. The low-temperature stages reside within a dewar with a main-shell temperature of about $260 \mathrm{~K}$ in orbit. Thus, the instrument aperture is a series of openings at temperatures spanning from $50 \mathrm{mK}$ to $260 \mathrm{~K}$. The main objective of the Aperture Assembly (ApA) is to block visible, infrared, and ultraviolet light via thin-film blocking

caroline.a.kilbourne@nasa.gov 
filters, while allowing $\mathrm{x}$-rays to pass through with minimal absorption. The ApA accommodates radiation-blocking filters (BF) and associated mechanical, thermal, and electrical hardware. The ApA prevents water vapor and other contaminants from collecting on the blocking filters or penetrating into the dewar, and the accommodation for filter heaters provides decontamination capability.

The SXS design derives considerable heritage from the design of the calorimeter-based X-ray Spectrometer (XRS) of Suzaku and Astro-E, and the starting points for the ApA and BF design for SXS were the similar subsystems of XRS ${ }^{5}$. The XRS design consisted of five aluminum-coated, polyimide, thin-film filters, with the two warmest filters closing out a cylindrical contamination barrier consisting of a gamma-alumina fiber-epoxy composite tube with a Ti foil liner. The two warmest filters were equipped with decontamination heaters. That basic system outline was preserved for SXS, but because of major differences in the cooling system, the temperatures and spacing of the intermediate filters were required to change. Additionally, progress in blocking-filter technology, specifically the use of silicon supporting meshes, was applied to the new mission to increase the strength of the larger filters, minimize the impact of localized damage to any of the thin films, and enhance the effectiveness of heating elements placed on the filter frames.

\section{HIGH-LEVEL SYSTEM DESIGN}

\subsection{Components}

There are a total of nine separate sub-components and sub-assemblies that compose the combined ApA and BF subsystem. The name of each is derived from the dewar interface to which it attaches mechanically and thermally. These interfaces are listed and defined in Table 1.

Table 1. Dewar interfaces of the SXS aperture assembly and blocking filters

\begin{tabular}{|l|l|l|}
\hline \multicolumn{1}{|c|}{ Interface } & $\begin{array}{c}\text { Standard } \\
\text { Abbreviation }\end{array}$ & Nominal Temperature \\
\hline detector enclosure and heat sink (Calorimeter Thermal Sink) & CTS & $50 \mathrm{mK}$ \\
\hline detector assembly structure & DA & $1.3 \mathrm{~K}$ \\
\hline Joule-Thomson cooler interface & JT & $4 \mathrm{~K}$ \\
\hline inner vapor-cooled shield & IVCS & $28 \mathrm{~K}$ \\
\hline outer vapor-cooled shield & OVCS & $150 \mathrm{~K}$ \\
\hline dewar main shell & DMS & $250 \mathrm{~K}$ \\
\hline
\end{tabular}

Starting from the base and progressing upward, they are as follows:

1. Calorimeter Thermal Sink Filter (CTSL-F): The CTSL-F closes out the detector enclosure. The CTS filter is permanently integrated into the CTS lid.

2. Detector Assembly Lid (DAL): The DAL is the top closeout to the DA, and also supports the DAL-FC (\#3) via an o-ring.

3. Detector Assembly Lid Filter/Carrier (DAL-FC): The DAL-FC supports the DAL filter. There are no active electrical or thermal components associated with the DAL-FC. The DAL filter and carrier are installed together but are never permanently integrated. For the purposes of this enumeration, however, the DAL filter will be considered part of the DAL-FC sub-assembly.

4. Joule-Thomson Baffle (JTB): The JTB is attached to the aperture in the JT Shield (JTS) and provides radiation and contamination baffles. 
5. Inner Vapor-Cooled Shield Mount (IVCS-M): The IVCS-M is attached to the IVCS and supports the IVCSFC (\#6), via an o-ring.

6. Inner Vapor Cooled Shield Filter/Carrier (IVCS-FC): The IVCS-FC supports the IVCS filter and associated mechanical, thermal, and electrical components.

7. Aperture Cylinder Sub-Assembly (ApC-SA): The ApC-SA consists of the Aperture Cylinder Tube (ApC-T), bonded to the OVCS Mount (OVCS-M) and to the DMS Mount (DMS-M). The OVCS-M supports the OVCSFC (\#7) via an o-ring. The ApC-T provides thermal isolation and a vapor barrier. The DMS-M supports the DMS-FC (\#8) via an o-ring. The ApC-SA is attached to the OVCS via thermal straps on the OVCS-M end, and is attached to the DMS at the DMS-M end directly, via an o-ring seal.

8. Outer Vapor-Cooled Shield Filter/Carrier (OVCS-FC): The OVCS-FC supports the OVCS filter and associated mechanical, thermal, and electrical components.

9. Dewar Main Shell Filter/Carrier (DMS-FC): The DMS-FC supports the DMS filter and associated mechanical, thermal, and electrical components.

Fig. 1(top) is a cross-sectional schematic of the SXS ApA. Note that the CTSL-F, DAL, JTB, IVCS-M, and the ApC-SA are not in physical contact, but are held in position by the different temperature stages. Thus, the ApA is integrated into the dewar one sub-assembly at a time and never constitutes an assembly by itself. Fig. 1 (bottom) is the same view, without the CTS, with the filter-carrier assemblies removed and shown separately to the side. The four filter carriers (FC) are installed after the dewar main shell is attached.

\subsection{Thermal design, analysis, and performance}

Thermal design drivers for the ApA included minimizing heat transfer between various dewar stages, meeting filter temperature requirements during normal operation, and providing for decontamination of the filters through heaters and interface design. The thermal design is complicated by the steep temperature gradients, the need to establish a hermetic seal, and the accommodations for decontamination heaters on the filters. Key features of the mechanical/structure and thermal design, shown in Fig. 1, are:

1. Thin-walled gamma alumina/titanium foil tube minimizes the thermal conductance between the DMS and the OVCS. The OVCS-M is recessed into the dewar far below the opening in the actual OVCS, to maximize the isolation; the free length of the tube is $74 \mathrm{~mm}$.

2. Heat sinking of the OVCS-M to the OVCS is achieved via three thermal straps made of high-conductivity copper.

3. Conductive breaks isolate the OVCS-M, the IVCS-M, JTB, DAL, and CTS.

4. Low emissivity coatings minimize radiation heat transfer. Nested contamination baffles also act as radiation baffles, minimizing radiative heat transfer between shield-mounted hardware.

5. Filter heaters on the DMS filter allow continuous heating of the filter to prevent absorption of organic contamination from the spacecraft.

6. Filter heaters on the OVCS and IVCS filters enable contingency decontamination of the filters to remove water ice.

7. Filter mounts provide low-conduction contacts to minimize heater power and dewar heat transfer during heater operation. 


\section{Aperture Assembly (ApA)}

Aperture Cylinder Sub-Assembly (ApC-SA)

- DMS-Mount (DMS-M)

- Aperture Cylinder Tube (ApC-T)

- OVCS-Mount (OVCS-M)

- Thermal Straps (TS)

Dewar Main Shell-Carrier (DMS-Car)

- DMS-Carrier Components

- DMS-Moisture Shields (DMS-MS)

- DMS-Filter (DMS-F)

Outer Vapor Cooled Shield-Carrier (OVCS-Car)

- OVCS-Carrier Components

- OVCS-Moisture Shields (OVCS-MS)

- OVCS-Filter (OVCS-F)

Inner Vapor Cooled Shield-Mount (IVCS-M)

Inner Vapor Cooled Shield-Carrier (IVCS-Car)

- IVCS Carrier Components

- IVCS Moisture Shields (IVCS-MS)

- IVCS Filter (IVCS-F)

Joule-Thomson Baffle (JTB)

Detector Assembly Lid (DA Lid)

Detector Assembly Lid-Carrier (DA Lid-Car)

- DA Lid Filter (DA Lid-F)

Calorimeter Thermal Sink Lid Filter (CTSL-F)

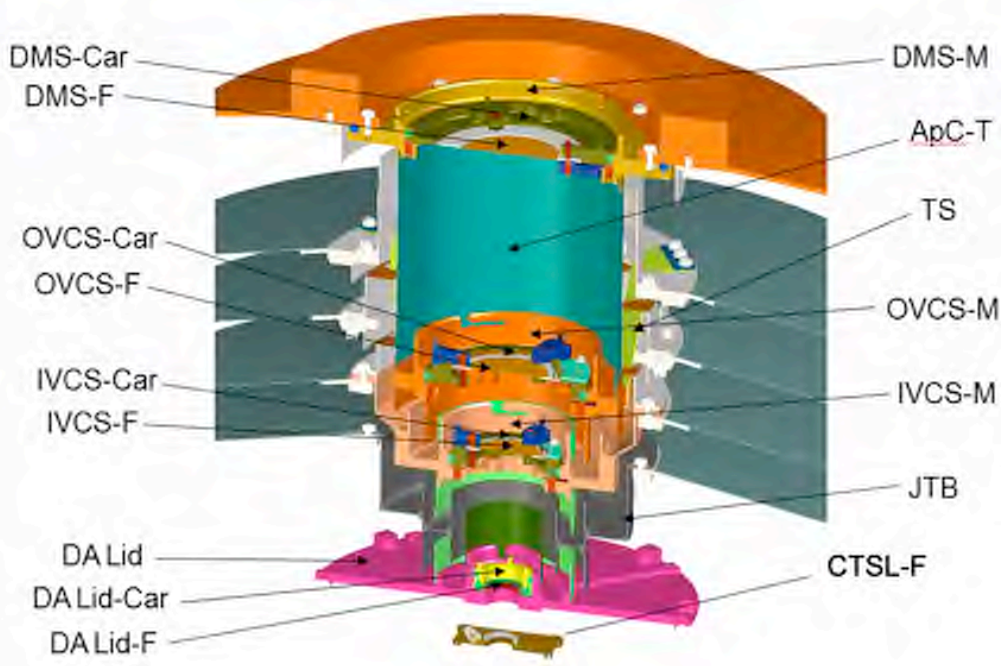

DALid-F

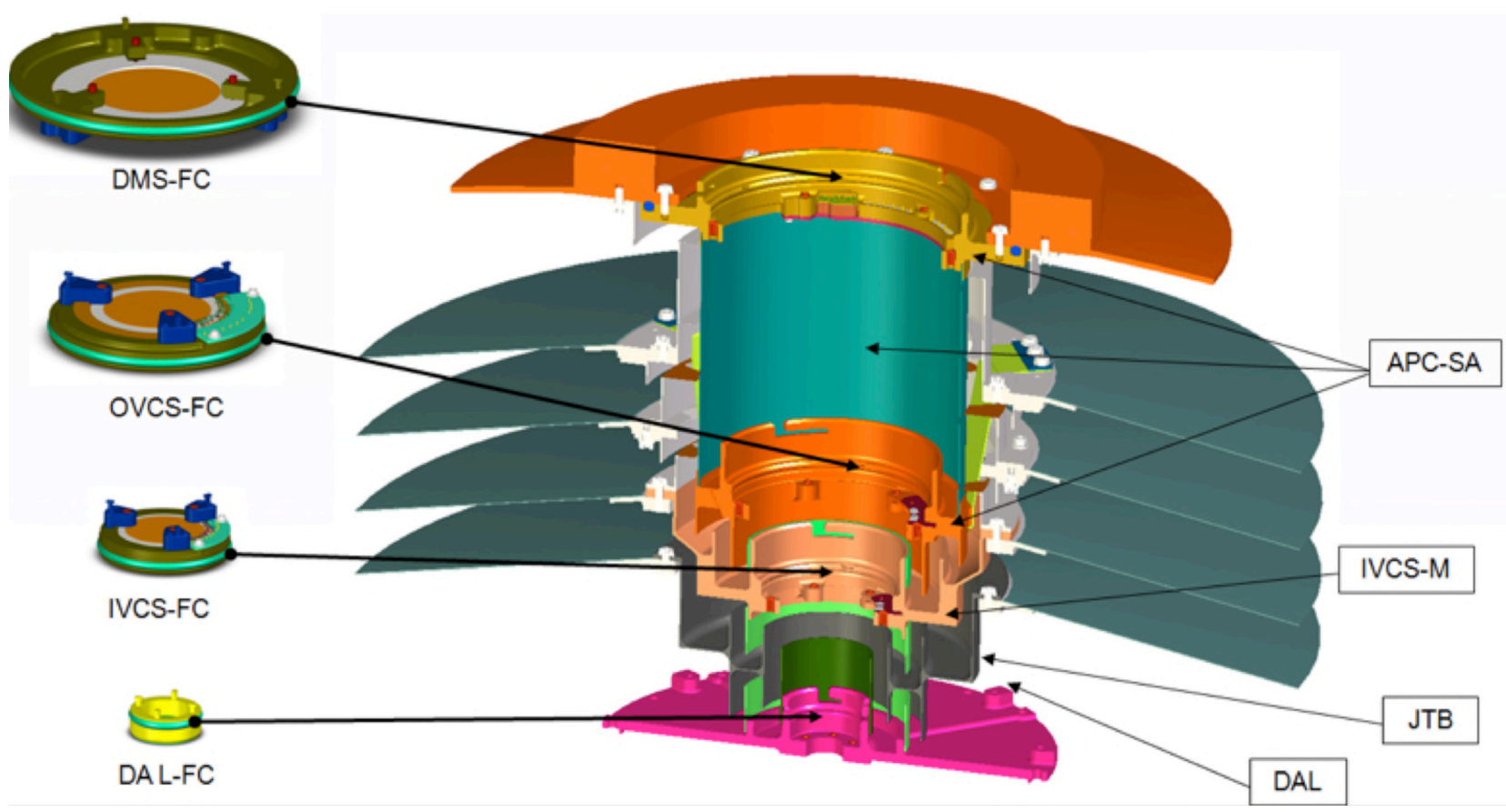

Figure 1. Schematic cross section showing the arrangement of the Hitomi/SXS Aperture Assembly and Blocking Filters. Top: with filter carriers installed and position of the fixed CTS filter shown. Bottom: with the filter carriers removed, and the CTS lid not shown. 
Table 2. Heat flows and temperatures for the SXS Aperture Assembly

\begin{tabular}{|l|l|l|l|l|l|}
\hline Interface & $\begin{array}{l}\text { In-flight } \\
\text { maximum } \\
\mathbf{( K )}\end{array}$ & $\begin{array}{l}\text { In-flight } \\
\text { minimum } \\
\mathbf{( K )}\end{array}$ & $\begin{array}{l}\text { Ground } \\
\text { minimum } \\
\mathbf{( K )}\end{array}$ & $\begin{array}{l}\text { Benchmark } \\
\text { Hot Case } \\
\mathbf{( K )}\end{array}$ & $\begin{array}{l}\text { Predicted Heat Flow into the } \\
\text { Dewar Shields for Benchmark } \\
\text { Hot Case (mW) }\end{array}$ \\
\hline DMS & 290 & 233 & 290 & 290 & N/A \\
\hline OVCS & 179 & 117 & 80 & 152 & $\begin{array}{l}710 \quad \text { (measured in subsystem } \\
\text { thermal tests) }\end{array}$ \\
\hline MVCS & 130 & 81 & 17 & 111 & 39 \\
\hline IVCS & 49 & 17 & 10 & 28 & 29 \\
\hline JT & 14 & 4 & 1.8 & 4 & 0.032 \\
\hline DA & 1.3 & 1.3 & 1.3 & 1.3 & 0.009 \\
\hline
\end{tabular}

The DMS and shield temperatures were projected to vary considerably during pre-launch testing and throughout the mission. The minimum shield temperatures occurred on the ground during liquid helium transfers, thus subsystem testing included these minima, with margin, as survival temperatures. The full range of flight and ground operation temperatures shown in Table 2, including margin (10K on DMS and $10 \%$ on cryogenic components), was used to verify the thermal design by analysis and to establish test ranges. The nominal hot case temperatures and the resultant heat flows are also shown. This case is important as it is used to determine He lifetime for the SXS mission. The heat load on the OVCS determined from subsystem thermal testing is included, while other heat flows shown represent the results from the thermal model (executed using the SINDA analysis package). This model was integrated into the dewar thermal model maintained by JAXA and Sumitomo Heavy Industries, the dewar developer. Radiative heat transfer and reflected energy along the exterior of the aperture cylinder are included in ApA thermal model.

Various thermal properties were measured during subsystem thermal-balance testing that are summarized in Table 3. The ApC-SA, DMS-FC, and OVCS-FC were tested together in a non-flight apparatus designed to simulate the flightdewar thermal interfaces and radiation environment. The IVCS-M and IVCS-FC were qualified down to $4 \mathrm{~K}$ (using the engineering model parts) and characterized for flight from a liquid nitrogen interface.

Table 3. Significant results of sub-system thermal balance testing

\begin{tabular}{|l|l|}
\hline Property & Value \\
\hline Thermal conductance of OVCS thermal straps at $150 \mathrm{~K}$ & $0.55 \pm 0.05 \mathrm{~W} / \mathrm{K}$ \\
\hline Heat load on 150 K OVCS from 290 K DMS & $0.71 \pm 0.02 \mathrm{~W}(0.56 \mathrm{~W}$ conducted and 0.15 W radiated) \\
\hline Heat load on 150 K OVCS from 240 K DMS & $0.37 \pm 0.02 \mathrm{~W}$ \\
\hline $\begin{array}{l}\text { Typical power to control DMS filter at } 320 \mathrm{~K} \text { when } \\
\text { DMS at } 290 \mathrm{~K} \text { and OVCS at } 150 \mathrm{~K}\end{array}$ & $0.38 \mathrm{~W}$ \\
\hline $\begin{array}{l}\text { Typical power to control DMS filter at } 320 \mathrm{~K} \text { when } \\
\text { DMS at } 240 \mathrm{~K} \text { and OVCS at } 150 \mathrm{~K}\end{array}$ & $0.68 \mathrm{~W}$ \\
\hline $\begin{array}{l}\text { Typical power to heat OVCS filter to } 170 \mathrm{~K} \text { when } \\
\text { OVCS at } 150 \mathrm{~K} \text { and DMS at } 290 \mathrm{~K}\end{array}$ & $\sim 0 \mathrm{~W}$ (filter is radiatively heated by DMS) \\
\hline Typical power to heat IVCS filter to $170 \mathrm{~K}$ from $90 \mathrm{~K}$ & $0.3 \mathrm{~W}$ \\
\hline
\end{tabular}




\subsection{Resonant frequencies}

The structural resonant frequencies of the ApA were assessed for each of its major components. A detailed finite element model and closed-form equations were used to identify minimum eigenvalue frequencies. Table 4 summarizes the minimum values, showing the analytical prediction and corresponding requirement. All predicted frequencies are axial (spacecraft $Z$ axis) except the ApC-SA, which is lateral. All results met project requirements.

Table 4. Fundamental frequencies of the SXS ApA sub-assemblies

\begin{tabular}{|c|c|c|}
\hline Component & $\begin{array}{c}\text { Predicted } \\
\text { Frequency (Hz) }\end{array}$ & $\begin{array}{c}\text { Minimum Frequency } \\
\text { Requirement (Hz) }\end{array}$ \\
\hline ApC-SA & 679 & 150 \\
\hline IVCS-M & 1505 & 150 \\
\hline JTB & 613 & 150 \\
\hline DA Lid & 388 & 100 \\
\hline FCs & 1424 & 150 \\
\hline Filters & 1333 & 150 \\
\hline
\end{tabular}

Due to the size and fragility of the filter and carrier assemblies, frequency measurements were not performed on these components. Results from cold vibration testing of the IVCS-M and FC assembly showed a minimum frequency of $1360 \mathrm{~Hz}$. This was scaled from the $\sim 80 \mathrm{~K}$ measurement temperature to the operational temperature of $\sim 20 \mathrm{~K}$. In ApCSA vibration tests, the minimum frequency was $831 \mathrm{~Hz}$.

\subsection{Thermo-mechanical testing}

A series of tests qualified the basic design features and verified the integrity of the flight units. Thermal and mechanical testing of the three silicon-mesh filters was done in their carriers in order to provide the appropriate mechanical and thermal interface. Operation of the heaters and thermometers required that the carriers be installed in mounts. These tests are summarized in Table 5. Note that vibration qualification of the ApC-SA with OVCS-FC and DMS-FC was done at room temperature, since the vibration dewar would cool the cylinder interface to $\sim 80 \mathrm{~K}$, introducing more thermal mismatch at the mount/tube interface than will be present at launch. Vibrating in this condition would be an over test. The under testing of a warm vibration was mitigated by separately measuring the strain at nominal operating temperatures and confirming the input to the analysis.

All ApA components that were launched on Hitomi passed their thermo-mechanical acceptance tests. Spare ApC-SA and IVCS-M units and DA and CTS filters also passed, but some damage was experienced on IVCS, OVCS, and DMS filters, which resulted in changing their ranking. These minor failures were mostly attributable to factors incidental to the tests themselves, such as from debris, pre-existing defects, or installation tools, as will be discussed along with other lessons learned in Section 7. 
Table 5. Thermo-mechanical tests of the SXS Aperture Assembly. FM = flight model; EM = engineering model

\begin{tabular}{|c|c|c|c|}
\hline Test description & $\begin{array}{l}\text { Temp. minimum } \\
\text { (of test) }\end{array}$ & $\begin{array}{l}\text { Temp. maximum } \\
\text { (of test) }\end{array}$ & $\begin{array}{c}\text { Number of cycles or } \\
\text { balance points }\end{array}$ \\
\hline $\begin{array}{l}\text { Warm vibration test of ApC-SA, DMS- } \\
\text { FC, and OVCS-FC (EM, FM, spares) }\end{array}$ & $295 \mathrm{~K}$ & $300 \mathrm{~K}$ & N/A \\
\hline $\begin{array}{l}\text { Thermal cycles of aperture tube bonded } \\
\text { between OVCS and DMS mounts }\end{array}$ & $80 \mathrm{~K}$ & $300 \mathrm{~K}$ & 5 cycles \\
\hline $\begin{array}{l}\text { ApC-SA Thermal Gradient / Thermal } \\
\text { Balance Test (EM and FM) }\end{array}$ & $117 \mathrm{~K}$ & $290 \mathrm{~K}$ & $3 \mathrm{DMS} / \mathrm{OVCS}$ temperatures \\
\hline $\begin{array}{l}\text { Thermal cycles of DMS FM and spare } \\
\text { carriers }\end{array}$ & $187 \mathrm{~K}$ & $298 \mathrm{~K}$ & $\begin{array}{l}\text { Primary and EM: } 3 \text { cycles } \\
\text { Spare: } 6 \text { cycles }\end{array}$ \\
\hline $\begin{array}{l}\text { Heater cycles of DMS FM and spare } \\
\text { carriers }\end{array}$ & $233 \mathrm{~K}$ & $320 \mathrm{~K}$ & $\begin{array}{c}10 \text { cycles } \\
(11 \text { for } \mathrm{EM})\end{array}$ \\
\hline $\begin{array}{l}\text { Thermal cycles of OVCS FM and spare } \\
\text { carriers }\end{array}$ & $<100 \mathrm{~K}$ & $298 \mathrm{~K}$ & $\begin{array}{l}\text { Primary: } 3 \text { cycles } \\
\text { Spare: } 6 \text { cycles }\end{array}$ \\
\hline $\begin{array}{l}\text { Heater cycles of OVCS FM and spare } \\
\text { carriers }\end{array}$ & $117 \mathrm{~K}$ & $170 \mathrm{~K}$ & $\begin{array}{c}10 \text { cycles } \\
(11 \text { for } \mathrm{EM}) \\
\end{array}$ \\
\hline $\begin{array}{l}\text { Thermal cycles of IVCS FM and spare } \\
\text { carriers }\end{array}$ & $80 \mathrm{~K}$ & $290 \mathrm{~K}$ & 5 cycles \\
\hline Thermal cycles of IVCS EM carrier & $9 \mathrm{~K} / 85 \mathrm{~K}$ & $292 \mathrm{~K}$ & $\begin{array}{l}1 \text { cycle to the lower } \\
\text { temperature, } 1 \text { to } 85 \mathrm{~K}\end{array}$ \\
\hline $\begin{array}{l}\text { Heater cycles of IVCS FM and spare } \\
\text { carriers }\end{array}$ & $80 \mathrm{~K}$ & $170 \mathrm{~K}$ & 20 cycles \\
\hline Heater cycles of IVCS EM carrier & $<28 \mathrm{~K}$ & $170 \mathrm{~K}$ & 12 cycles \\
\hline $\begin{array}{l}\text { Cold vibration test of IVCS (EM, FM, } \\
\text { spares) in mount }\end{array}$ & $80 \mathrm{~K}$ & $80 \mathrm{~K}$ & N/A \\
\hline Thermal cycles of DA and CTS filters & $4 \mathrm{~K}$ & $295 \mathrm{~K}$ & $\begin{array}{l}4 \text { cycles (qualification) } \\
1 \text { cycle (acceptance) }\end{array}$ \\
\hline $\begin{array}{l}\text { Lifetime Test Unit (EM spare OVCS } \\
\text { FC) thermal cycles }\end{array}$ & $85 \mathrm{~K}$ & $290 \mathrm{~K}$ & 25 cycles \\
\hline $\begin{array}{l}\text { Lifetime Test Unit (EM spare OVCS } \\
\text { FC) heater cycles }\end{array}$ & $90 \mathrm{~K}$ & $170 \mathrm{~K}$ & 12 cycles \\
\hline
\end{tabular}

\section{FILTER ASSEMBLIES}

\subsection{Thin-film filters and their support structures}

Low temperature $\mathrm{x}$-ray microcalorimeters require very thin filters for rejecting non-x-ray radiation while providing the highest possible transmission in the $\mathrm{x}$-ray band $(0.1-10 \mathrm{keV})$. Obtaining high $\mathrm{x}$-ray transmission requires films that are only about $1000 \AA$ thick, composed of polymer film coated with aluminum. These filters have diameters that can be as large as several centimeters and are extremely delicate. The use of these filters in the ultra low temperature environment required by the SXS is even more challenging since the filters can become tight when under vacuum (due to moisture loss) and very brittle when cold.

The BF prevent radiation with wavelengths longer than x-rays from reaching the detector, where it would heat the pixels or induce photon shot noise. The filters also close out various thermal shields to minimize heat loads on the cryogens. For the entire stack of filters, the required maximum out-of-band transmission of the filters was 0.006 at $40.8 \mathrm{eV}$ (geocoronal He II), 0.02 at $21.2 \mathrm{eV}, 0.002$ at $10.2 \mathrm{eV}$, and $5 \times 10^{-9}$ in the IR range (3-30 microns). (The requirement for attenuation of the geo-coronal line was satisfied by the telescope thermal shield, with no additional requirement on the filters.) The required minimum in-band transmission for the stack was $16 \%$ at $0.6 \mathrm{keV}, 52 \%$ at $1.0 \mathrm{keV}, 70 \%$ at $6 \mathrm{keV}$, and $70 \%$ at $10 \mathrm{keV}$. The attenuation requirements were met by design and measurements from the XRS programs, and 
the white-light transmission of each filter was measured to assess pinhole transmission. The in-band transmission requirements were verified with $\mathrm{x}$-ray measurements.

The SXS filters use aluminized polyimide and are highly reflective in the visible and IR. The UV attenuation is determined by both the aluminum and polyimide thicknesses. The chosen thicknesses came from a trade-off of the highthroughput at low energies, margin in the thermal design and detector noise budget, and mechanical strength. Strength is required to survive numerous thermal cycles to low temperatures, mechanical vibration, and pressure gradients of a few hundred $\mathrm{Pa}$. The outer filter was also required to limit the integrated diffusion of helium into the dewar to $<10^{-7} \mathrm{~Pa}$, given a required helium pressure in the spacecraft of $<10^{-6} \mathrm{~Pa}$. The nominal thicknesses of the films used in the SXS filters are tabulated in Table 6 . Results of the calibration of the filter transmissions are reported in Eckart, et al. ${ }^{6}$

The CTS and DA filters had Al frames and were procured from Luxel Corporation as integrated units. For the DMS, OVCS, and IVCS filters, the silicon-mesh frames were provided by the University of Wisconsin, the aluminized polyethylene (PET) close-outs (discussed in Section 3.3) were produced at Goddard, and the thin-film filter material was supplied by Luxel. Luxel integrated their thin films with the support frames and close outs at their facility. Specialized tooling and bake-out procedures were developed by Luxel to optimize contact between the filter film and the silicon grid. The development of the mesh filters was achieved via the collaboration of these three institutions.

The University of Wisconsin developed the monolithic two-level silicon support meshes for the three larger filters. Each mesh contains a coarse hexagonal grid that is $0.2 \mathrm{~mm}$ thick. Within each $5.28 \mathrm{~mm}$ wide coarse hexagonal cell is a fine mesh that is either $0.025 \mathrm{~mm}$ (IVCS, OVCS) or $0.008 \mathrm{~mm}$ (DMS) thick. The layout of the $0.33 \mathrm{~mm}$ fine-mesh hexagons is the same in each complete coarse-mesh hexagon, independent of filter type. Fig. 2 shows the coarse and fine mesh designs. A backing ring of $0.4 \mathrm{~mm}$ thick $\mathrm{Si}$ is glued to each mesh, and the kinematic-mount contact features are etched into the backing ring. Fig. 7 is a photo of the OVCS filter that clearly shows the two-level mesh structure.

The basic mechanical performance is quite good: on the $350 \mu \mathrm{m}$ pitch of the hexagonal front mesh, $45 \mathrm{~nm}$ polyimide films will withstand more than $6000 \mathrm{~Pa}$ pressure differences. The open-area fraction of each two-level structure is over $95 \%$, and the Si becomes transparent at the top of the SXS bandpass. The meshes are constructed from standard siliconon-insulator (SOI) wafers that are produced commercially by bonding two wafers together with an oxide layer, then grinding each side down to the desired thickness. These wafers are procured with the desired thicknesses for the front and backing meshes, and one mesh is patterned and etched from each side with a deep reactive ion plasma etch (DRIE) that can produce a vertical profile and stops on the oxide layer between the wafers. The oxide is then removed from the mesh openings with an acid etch. The silicon structure is completed by gluing a silicon backing ring containing the kinematic-mount interfaces.

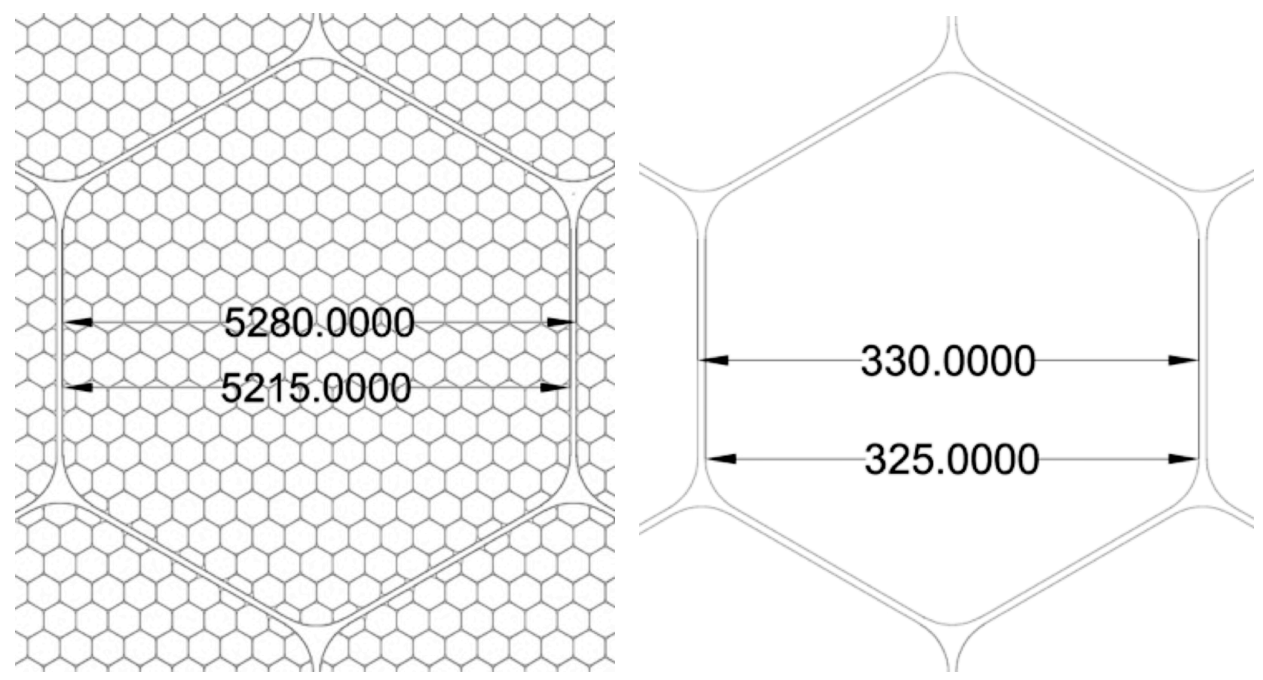

Figure 2. Dimensions (in microns) of the coarse mesh (left) and fine mesh (right) cells of the DMS, OVCS, and IVCS blocking filters of SXS 
Table 6. Specified thicknesses of the thin films used in the SXS blocking filters

\begin{tabular}{|l|l|l|}
\hline Filter or close-out & Aluminum Layer & Polymer Film \\
\hline CTS and DA & $50 \mathrm{~nm}$ & $75 \mathrm{~nm}$ (polyimide) \\
\hline IVCS, OVCS, and DMS & $100 \mathrm{~nm}$ & $100 \mathrm{~nm}$ (polyimide) \\
\hline Close-out (moisture shield) & $30 \mathrm{~nm}(\mathrm{x} 2$ sides) & $6000 \mathrm{~nm}$ (PET) \\
\hline
\end{tabular}
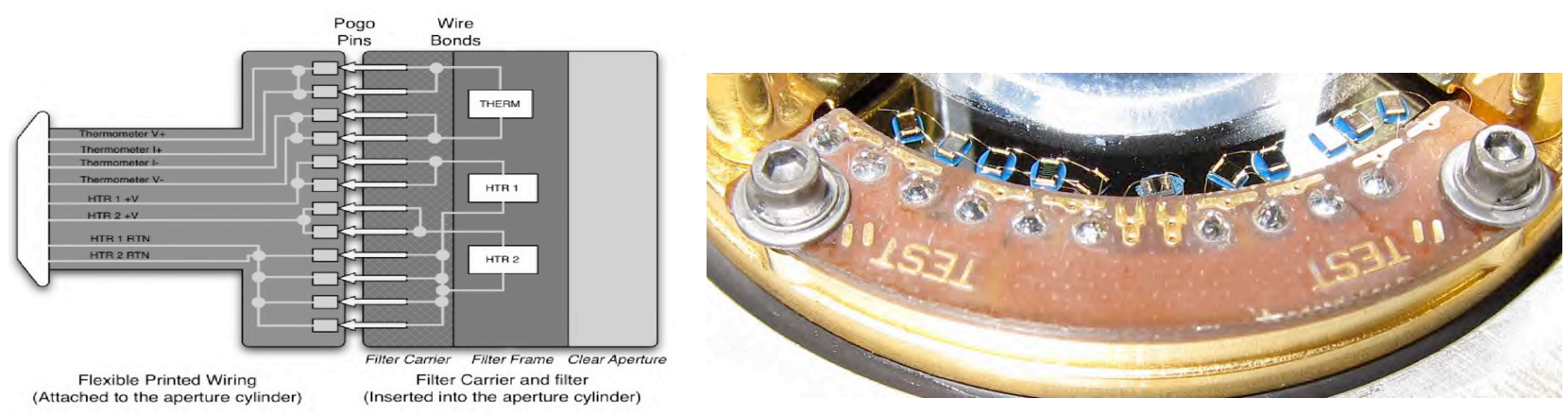

Figure 3. Electrical schematic (left) of the filter thermometer and heater wiring of a mesh filter and photograph (right) of heater and thermometer components on the IVCS filter.

\subsection{Filter heaters and thermometers}

The ApA electrical accommodations include heaters and thermometers on the DMS, OVCS, and IVCS filters. Each of these filters has a thermometer and two separate heater circuits for redundancy. Each heater circuit consists of several $600-\Omega$ microelectronic resistors in parallel. The thermometer and heaters were bonded to the filter frame via epoxy prior to application of the thin film. Gold wire bonds connect the components to each other and to a printed wiring board. The wire-bonding configuration also provides redundancy for both the heater and thermometer circuits, allowing full functionality even if one bond on the positive side and one bond on the negative were to fail. The DMS resistors also feature double bonds between the series components. Connections between each FC and its mount are provided by spring-loaded pins, which are engaged when the FC is installed into its mount. All connections are made through two spring-loaded pins in parallel, for redundancy. Fig. 3 shows the schematic of the redundant circuitry and a photo of the heaters and thermometers on the frame of the IVCS filter.

\subsection{Filter carriers and close-outs}

All filters except the CTS are installed after the dewar main shell is attached. Each of these filters requires a carrier to provide the mechanical, thermal, and electrical interface (for IVCS, OVCS, and DMS) between the filter and its mount and the mechanical interface to the installation tool.

The DAL-FC is made from aluminum and coated in gold. The DA blocking filter does not have any electrical or thermal features. The DA filter is simply sandwiched between a set of ball plungers and an o-ring and does not have any rotational alignment requirements. Unlike the other filters, the DA filter and its carrier do not become permanently integrated with each other. Fig. 4 shows the CTS and DA filters. 

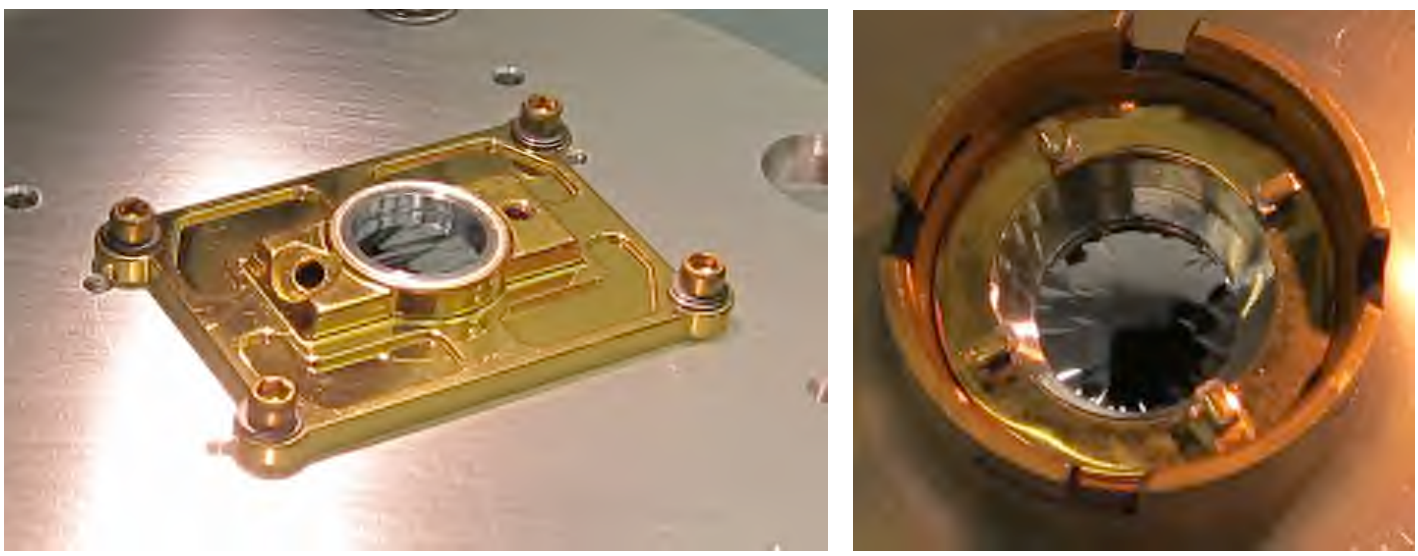

Figure 4. CTS lid with filter (CTSL-F, at left) and DAL filter and carrier (DAL-FC) in non-flight holder (right)
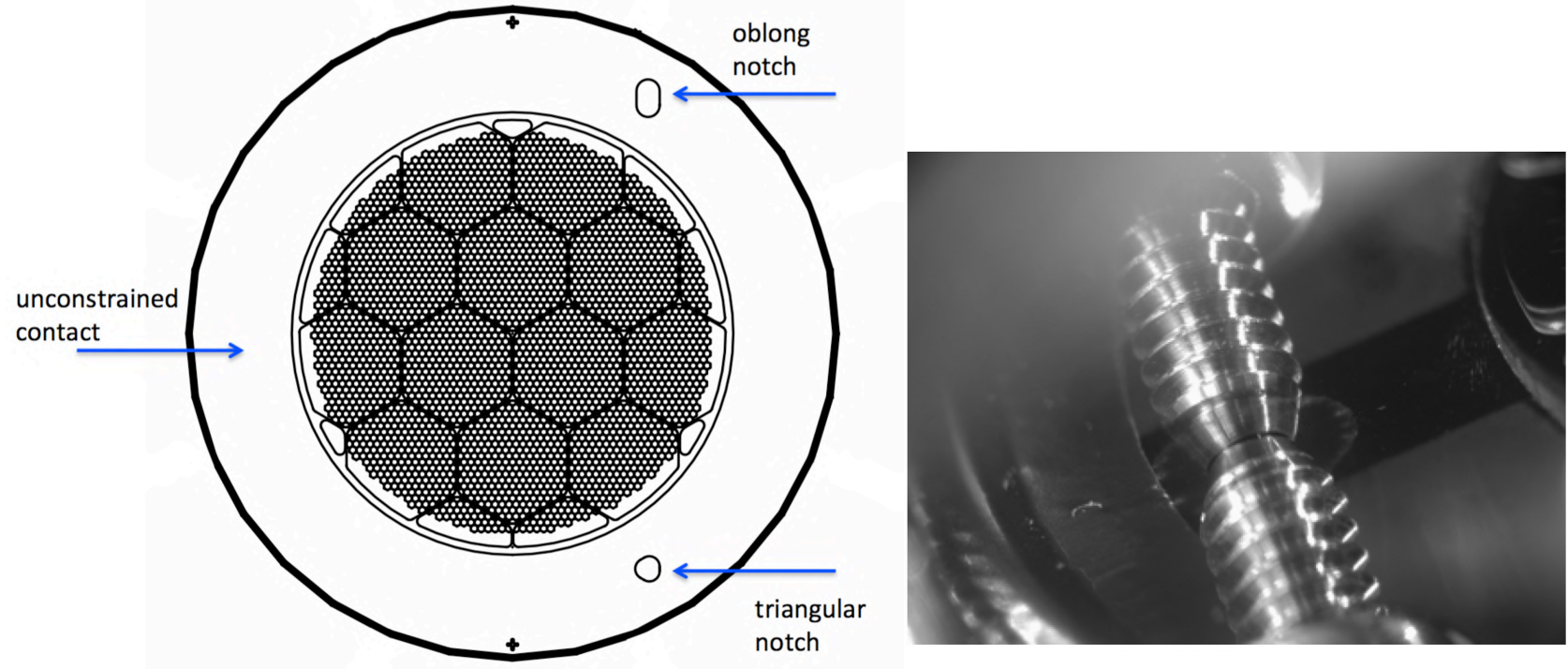

Figure 5. Left: kinematic mount points on the back of the IVCS filter. Right: photograph of a triangular notch resting on a ball-end screw. The reflection of the ball-end screw is seen in the metalized surface of the silicon frame.

The IVCS, OVCS, and DMS carrier rings are also made from aluminum and coated in gold. Each carrier has a circumferential o-ring that secures it into its respective mount. Additionally, six ball plungers in the mount support the carrier and adjust it to its proper height. Each blocking filter is supported in its carrier via a kinematic-mount system that consists of three ball plungers and three ball-end Ti set screws. Each pair of a ball-end screw and a ball-headed spring plunger supports the filter and engages with its kinematic mount features to constrain motion in 3 (triangular notch), 2 (oblong notch) or 1 (flat contact) degrees of freedom. The kinematic mounting allows for free movement upon differential thermal expansion and contraction. Fig. 5 contains a drawing showing the kinematic mount points on the back of the IVCS filter and a photograph of a triangular notch resting on a ball-end screw. The kinematic-mount notches are laser cut/chemically smoothed through-holes. 


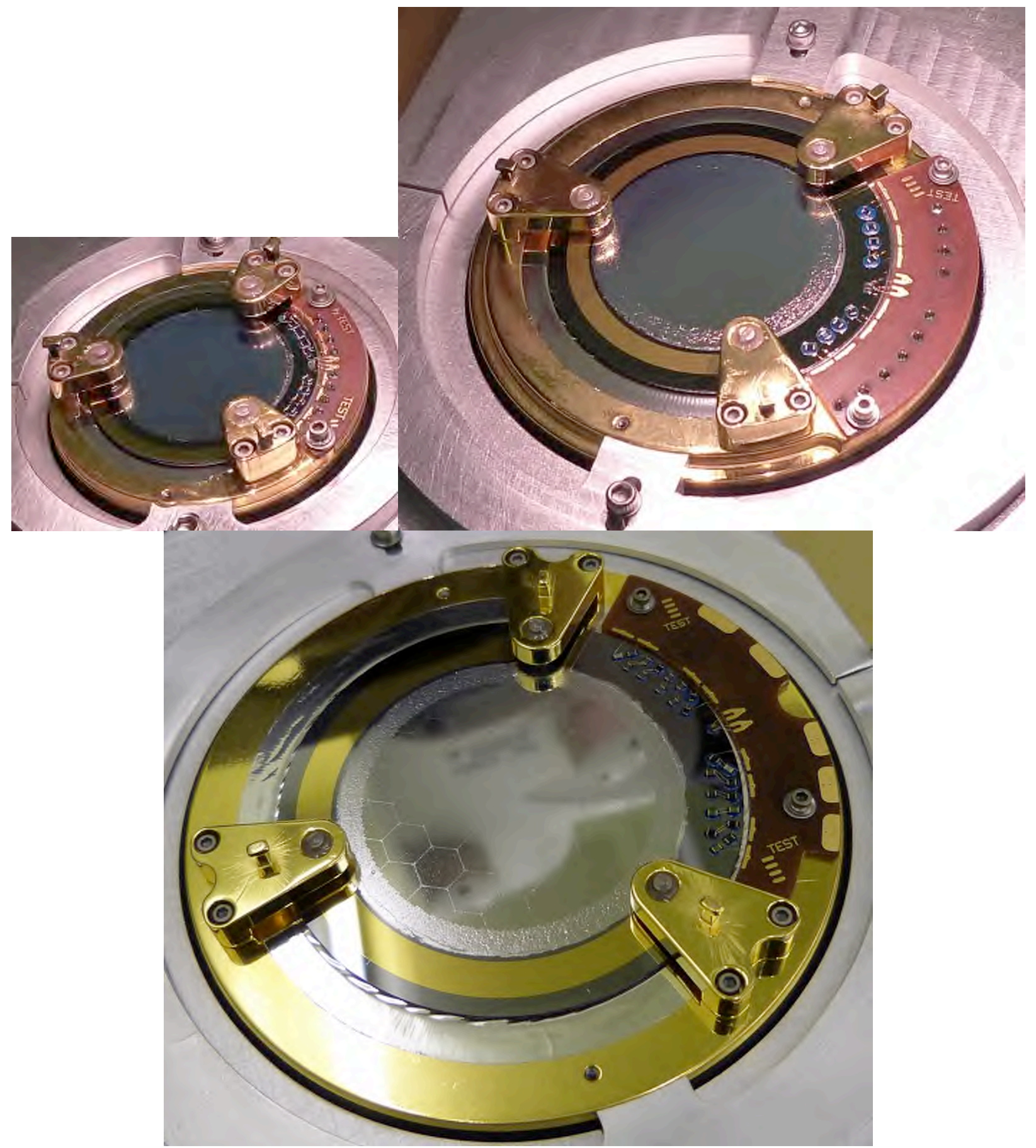

Figure 6. The IVCS (upper left), OVCS (upper right) and DMS (bottom) filter carriers for SXS. The three triangular structures in each assembly hold the spring-loaded ball plungers.

On the IVCS and OVCS FCs, the structures that hold the ball plungers in position also have T-shaped features that are grabbed by the installation and extraction tools. For the DMS carrier, which is inserted with the backing-ring side towards the tool, these pick-up features are on the back of the carrier ring. (The OVCS and DMS filters are installed with the lowest emissivity sides facing the inside of the ApC-SA.) The DMS ball-plunger structures also have pick-up features, for easier handling during assembly. 
The small gap between the filter frame and the carrier is covered by a Moisture Shield (MS) that is also referred to as a close-out. The MS is bonded to the filter frame at its inner diameter and to the carrier at its outer diameter. The MS is made of aluminized PET and incorporates a flexible feature to allow for thermal expansion and contraction of the silicon frame with respect to the aluminum carrier ring. This strain-release feature was formed into the aluminized PET sheet by a heated stainless steel mold. The MS blocks the passage of light, water vapor, helium, and other contaminants around each filter. Acceptable white-light transmission and helium permeation were verified on samples of the MS material.

The IVCS and OVCS carriers include a printed wiring board (PWB) with spring-loaded contact pins. Wire bonds electrically connect thermometers and heaters to the PWB. The spring pins contact the electrical contact pads in the mount when upon installation. The DMS is similar, but the spring-loaded pins are on the mount side of the interface.

Fig. 6 shows the DMS, OVCS, and IVCS FCs. Fig. 7 shows the back of the OVCS FC while held in the installation tool. This view clearly shows the two-layer mesh, the backing ring, the kinematic mounting points, the electrical contacts, and the MS interfaces. The equivalent features of the DMS and IVCS filters are conceptually similar.

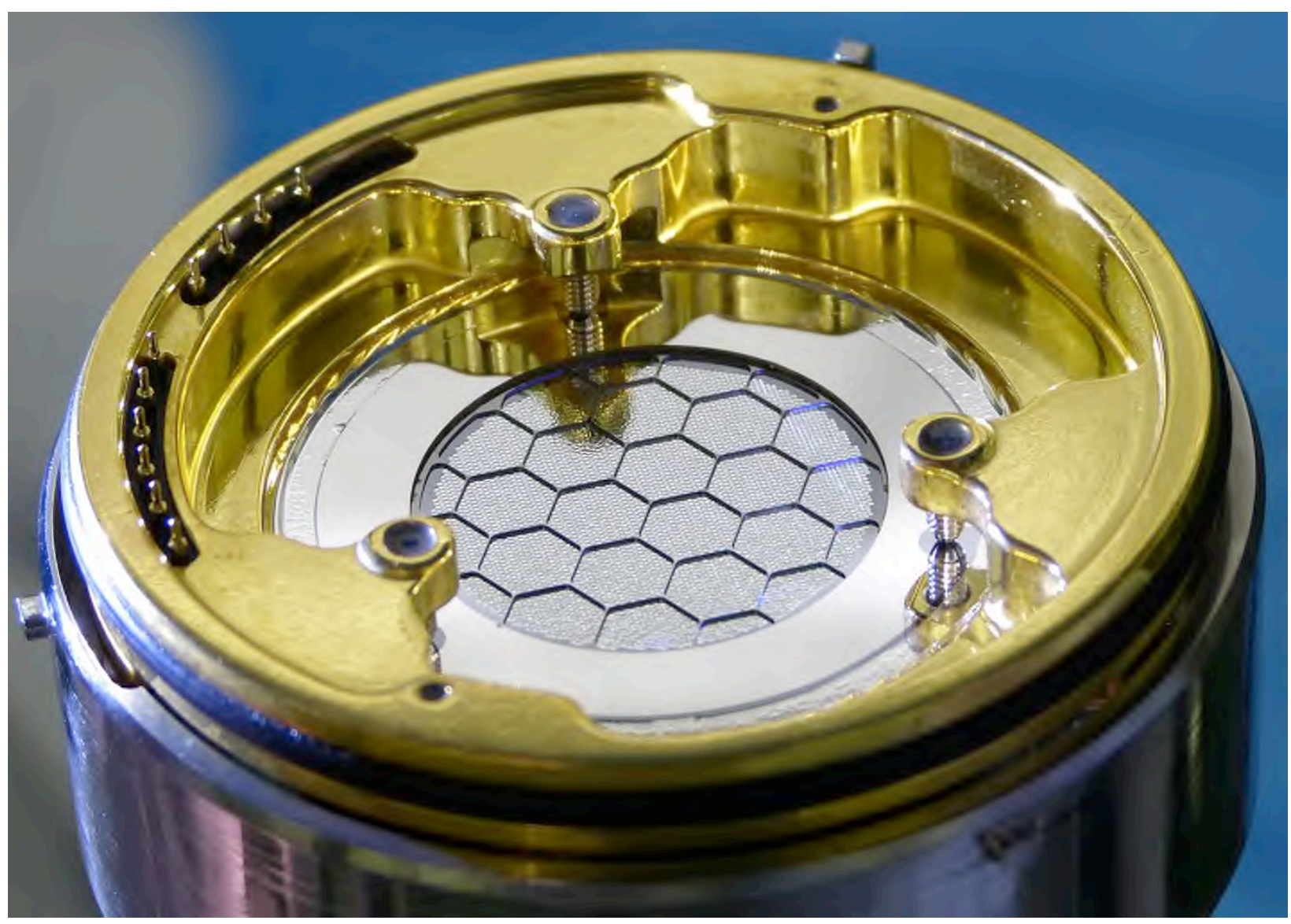

Figure 7. Photograph of FM OVCS Filter from the back, revealing the coarse and fine silicon mesh cells, the moistureshield interface to the silicon frame, the kinematic mounting, and the spring-loaded electrical contacts.

\subsection{DMS filter heating}

As a result of projected outgassing rates on the spacecraft, various contamination-mitigation schemes were pursued. The solution chosen was to keep the DMS filter temperature continuously controlled at an elevated temperature after the dewar gate valve was opened. The choice of control temperature for the DMS filter depends on an assessment of the most likely contaminants and what the constituents of the filter can safely tolerate. From analysis of spacecraft outgassing, DEHP $\left(\mathrm{C}_{24} \mathrm{H}_{38} \mathrm{O}_{4}\right)$ was assumed to be the dominant contaminant, based on the energy-dependent absorption 
associated with the contamination that accumulated on the Suzaku XIS filters. Measurements of accumulated contamination during baking of the Suzaku spacecraft indicated that the sticking coefficient was zero for surface temperatures $>279 \mathrm{~K}$. To allow for uncertainty in the nature of the major contaminant, the dependence on surface properties, and the uncertainty in thermal modeling, the plan established for DMS-filter heating was to maintain all points on the DMS filter at a temperature $>300 \mathrm{~K}$.

The filter temperature is limited by the adhesive used in the construction of the filters. Luxel originally advised limiting the filter temperature to $<65{ }^{\circ} \mathrm{C}(338 \mathrm{~K})$, but that was a standard limit based on an adhesive that was no longer in use at the time of the construction of the SXS filters. Later Luxel revised the recommended limit to $100{ }^{\circ} \mathrm{C}(373 \mathrm{~K})$. A test of the spare EM DMS filter showed that the filter could be heated over $420 \mathrm{~K}$ without introducing any permanent change to the appearance.

The operating plan was to control the temperature of the DMS filter frame at $320 \pm 2 \mathrm{~K}$. The expected required heater power ranges from $0.66 \mathrm{~W}$ at the coldest main-shell temperature of $233 \mathrm{~K}$ to $0.36 \mathrm{~W}$ at a $295 \mathrm{~K}$ main-shell temperature, based on subsystem-level testing.

Due to the low thermal conductivity of the silicon mesh and radiative losses from the thin film, the DMS filter will have a gradient from the control thermometer to the center of the mesh in addition to a gradient around the filter frame from the heater/thermometer to $180^{\circ}$ opposite. SINDA modeling showed that a cold-case DMS temperature of $233 \mathrm{~K}$ results in the highest heater power $(850 \mathrm{~mW})$ and therefore the highest gradient, approximately $23 \mathrm{~K}$. The coolest area is slightly offset from the center. A lower gradient was projected for the hot case, due to the reduced heater power $(400 \mathrm{~mW})$.

Given that the model predicted a higher power to control the filter temperature than experimentally determined, we sought a secondary method to increase our confidence that the actual temperature gradient across the filter would be acceptable A simple physical model was developed to check the SINDA results and fold in actual measurements. This model addresses the radial gradient in the thin film from radiative losses separately from the lateral gradient in the thicker silicon frame of the filter. This model yielded a value for the conductance of the filter to the carrier that was consistent with the heater power needed for both a $295 \mathrm{~K}$ and $265 \mathrm{~K}$ main shell. This conductance in turn showed that the gradient in the filter frame for the cold case would be $\sim 12 \mathrm{~K}$. The gradient within the thin film depends on its emissivity. Using a conservative emissivity of 0.2 , the coldest point in the filter would be $16 \mathrm{~K}$ lower than its perimeter, assuming a uniform temperature at the boundary. Using a more realistic emissivity, the gradient in the frame will dominate the gradient in the film. This simple model shows that conservative assumptions reproduce the scale of the gradients determined in the SINDA modeling.

There are additional layers of conservatism. The cold case represents an extreme DMS temperature that is lower than the expected minimum. Additionally, the plan to keep all point of the DMS filter above $300 \mathrm{~K}$ includes margin over the minimum temperature of $279 \mathrm{~K}$. Thus we had confidence that the filter can be maintained at an adequate temperature.

\subsection{OVCS and IVCS filter heating}

The OVCS, and IVCS filters are cold enough to condense water vapor that is trapped and released slowly by the multilayer-insulation thermal blankets within the cryostat. The DA filter and the CTS filter are also cold enough to condense water vapor but they are fully enclosed within cold shields that do not allow the penetration of water vapor. The primary mitigation for water condensation on the OVCS and IVCS filters is the water vapor barrier in the aperture cylinder and baffling. The secondary mitigation is a heater and a corresponding thermometer to monitor the filter frame temperature during operation.

The desorption rate of water is effectively zero below $140 \mathrm{~K}$ and increases rapidly above $150 \mathrm{~K}$. The sticking probability of water on a cold surface is unity in this temperature range. According to Speedy et al. (ref. 7), increasing the temperature to $170 \mathrm{~K}$ results in a desorption rate of $6-12$ monolayers per second, depending on the morphology of the ice. Therefore, if ice accumulation were to have become evident in orbit, the IVCS and OVCS filters would be heated to $170 \mathrm{~K}$. 


\section{DETECTOR-ASSEMBLY LID, FILTER MOUNTS, APERTURE CYLINDER, AND JOULE-THOMSON BAFFLE}

\subsection{Mounts and baffles}

As can be seen in Fig. 1, the ApA components from the OVCS to the DA are arranged with interleaving baffles. These baffles ensure that no molecular contamination has a direct line of sight to a filter, but must first encounter a cold baffle surface to which it will stick.

The main bodies of all of the aperture components are made of gold-plated aluminum. Surfaces engaging the installation tool are anodized. The filter receptacles in the DA lid and the IVCS, OVCS, and DMS mounts all support their respective filter via an o-ring groove and six axial ball plungers. Rotational alignment is not required for the DA filter, but for the others, a radial slot in the mount provides a visual reference for the rotational alignment. (The alignment is actually achieved via the installation tool, which makes use of an alignment tab on the carrier.) Circumferential tabs on the installation tool engage slots around each filter receptacle. As the tool pushes the carrier into place, the force is reacted at the tabs.

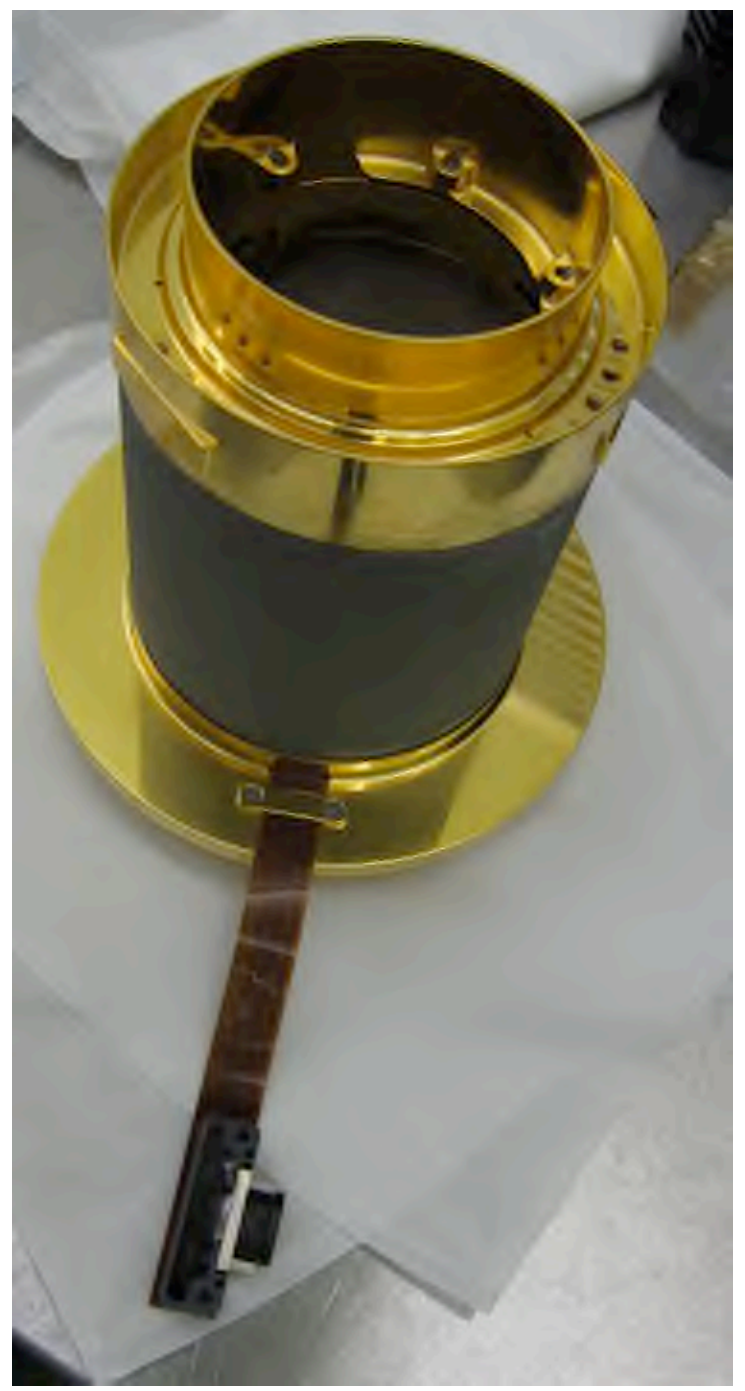

Figure 8. SXS Aperture Cylinder sub-assembly, OVCS side up. The harness seen in this view is for the DMS. 
The IVCS and OVCS mounts each support a flat trace harness that terminates on a connector at one end and on a PWB with gold-plated contacts on the other; for the DMS, the PWB contains spring pins. The PWB is located radially and below the o-ring groove in the mount. When the carrier is pressed into position, the spring-loaded pins align with the contacts and provide electrical continuity from the filter thermometer and heaters to the connector.

\subsection{Aperture Cylinder}

The aperture cylinder consists of a two-ply gamma-alumina fiber-epoxy composite with a Ti foil liner that is co-cured into the tube. The Ti foil is alloy 15-3-3-3 and is $0.0127 \mathrm{~mm}$ thick. The foil is considered the primary contamination barrier. Due to design heritage from XRS, a water permeation test was not performed on either a qualification unit or flight unit of the aperture cylinder. Since water will pass through the Ti only through cracks or pinholes, and not diffusion, a light-leak test was performed as a means of verifying the water permeation requirement. Fig. 8 is a photograph of the ApC-SA.

To minimize the pressure differential across the filters to 1 Torr (133 Pa) or less during (i) dewar pumpdown and (ii) filter installation, a set of venting holes were placed in the OVCS Mount. Without these, the ApC-SA would have been a completely enclosed volume. Three 1-mm diameter holes are positioned 120 degrees apart. These have a collective cross-sectional area of $2.36 \mathrm{~mm}^{2}$ against a total enclosed ApC air volume of $4.73 \times 10^{5} \mathrm{~mm}^{3}$. For a 20 Torr/min dewar pump down, a 1 Torr differential across the filters only requires a vent area of $0.005 \mathrm{~mm}^{2}$, which is equivalent to a $174 \mathrm{x}$ area margin. As for filter insertion, a $20 \mathrm{~mm} / \mathrm{s}$ installation velocity (much faster than the procedure) would require a $0.006 \mathrm{~mm}^{2}$ vent area, which has an equivalent margin of $135 \mathrm{x}$.

\section{INTEGRATION INTO THE DEWAR}

The separate components of the ApA were integrated into the dewar as the shields and associated multi-layer insulation and harnesses of the top (aperture side) of the dewar were built up. The JTB, IVCS-M, and the ApC-SA (OVCS side) each mounted to an adjustable interface ring in its respective shield. The dewar main shell position was pinned. It was the responsibility of JAXA to align the helium tank and shield apertures to the dewar reference. The alignment of the detector and the CTS and DA apertures is discussed in Chiao et al. ${ }^{8}$ The JTB, IVCS-M, and the ApC-SA did not use alignment pins. The ApA alignment plan largely relied on having adequate margin in the root-mean-square sum of all the contributions to the clear-aperture budget. The clear aperture at the IVCS had the smallest margin, so a method of locational biasing (use of temporary fasteners of calibrated shank diameter) was readied for usage pending receipt of the alignment report for the IVCS interface ring. Based on the report, it was decided that no bias was needed.

The dewar was inverted for the installation of the FCs, to mitigate the risk of particles or objects falling through the filters. The filter installation tools were required to support and clamp each FC during installation. The tool required a capability to install primarily by feel as visibility was limited except for the DMS-M. The outer head of each tool contained tabs that temporarily engaged slots on the FC mounts to provide a reacting force while the inner shaft/head was inserted the FC into the mount. This process required a lever with enough mechanical advantage to ensure a quasistatic insertion of the FC until the o-ring seated into its groove in the mount, as the force of insertion was measured as high as $280 \mathrm{~N}$ in mock-ups of DMS-FC installation. This process was required to be completely repeatable and with essentially no mechanism lash shock introduced as the o-ring relaxed as it expanded into its seated location in the mount. Extensive attention to the design and manufacturing tolerance at each interface of the mechanisms, coupled with several design iterations and numerous rehearsal test runs, validated the design and resulted in the flawless installation of the flight FC. Fig. 9 is a photograph of the OVCS-FC being raised into the dewar for installation. The installation tool is shown supported and guided by an additional mechanism designed to improve control of the tools during installation.

\section{IN-FLIGHT PERFOMANCE}

Due to the premature end of the Hitomi mission, very little in-flight performance data was accumulated on the ApA subsystem. The superb early performance of the detectors ${ }^{9}$ indicated that the filters survived launch. The DMS, OVCS, and IVCS filters passed their electrical and thermal functional tests, reprising the results of ground tests. Continuous DMS temperature control had not yet started at the time of mission loss, but it was controlled for two orbits as part of the checkout. The DMS filter frame was controlled at $320 \mathrm{~K}$ while the main shell oscillated between $254 \mathrm{~K}$ and $259 \mathrm{~K}$. The average power required was $0.48 \mathrm{~W}$, consistent with extrapolation of results from ground testing. 


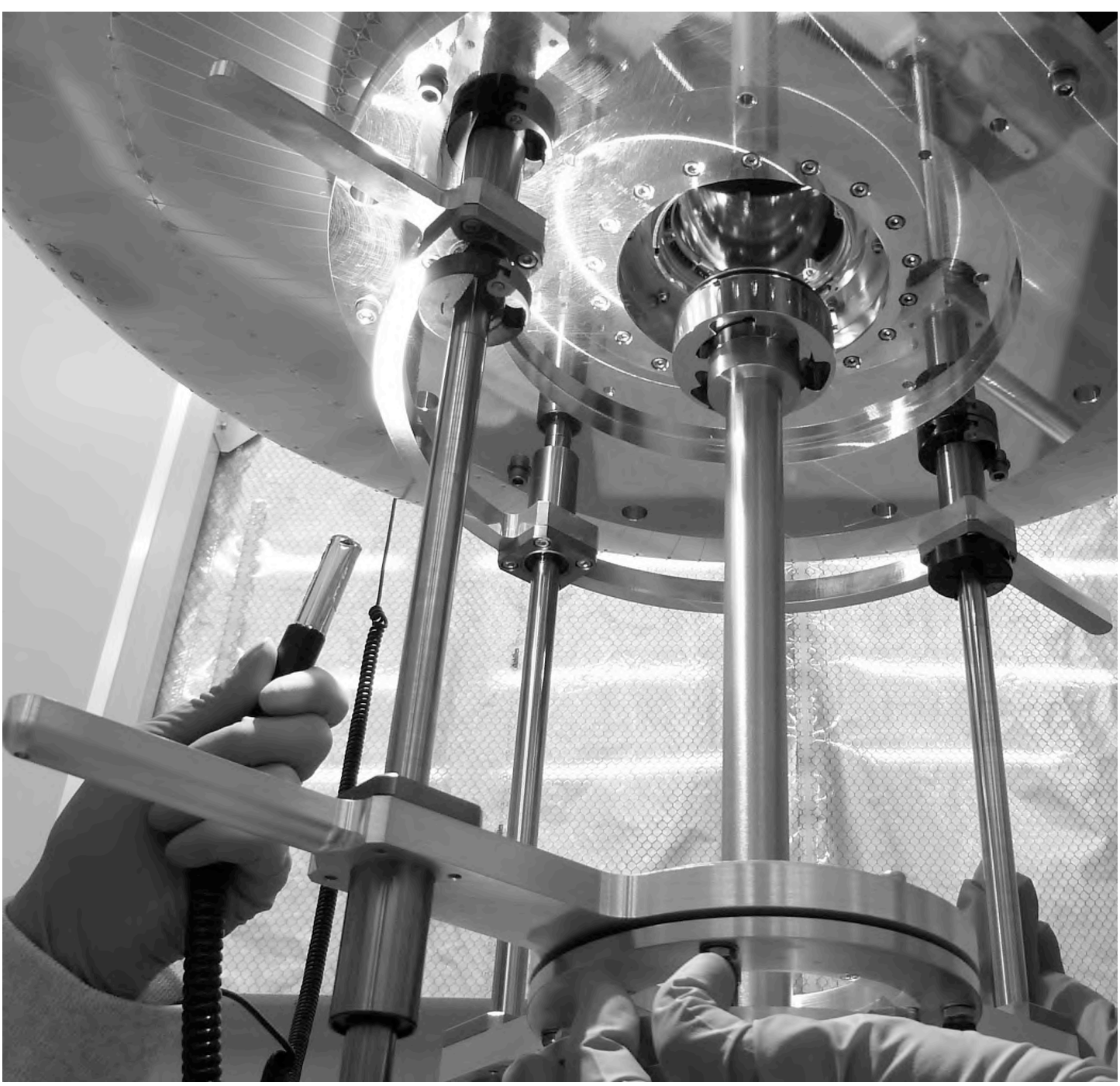

Figure 9. Photograph of the OVCS-FC being raised into the SXS dewar for installation. The installation tool is supported and guided by a mechanism attached to the dewar.

\section{LESSONS LEARNED}

The thin films of the blocking filters are susceptible to breakage by particles accelerated by test and launch vibration. Throughout the project, management of particles down to the required scale of $50 \mu \mathrm{m}$ was a continuous preoccupation. Frequent inspections with good lighting and optics were necessary. In several cases, inadequate cleaning at the early stages of component integration resulted in more difficult cleaning operations at a higher level of assembly. In the future, to the extent allowed by the thermal design, pockets that are difficult to inspect and access with cleaning tools are to be avoided.

The DMS, OVCS, and IVCS FCs were extremely difficult to assemble. Future implementations should strive to make the hand assembly easier and of lower risk to the thin films and fragile silicon meshes. We have identified a large number of improvements that could be made to the design of the silicon meshes, the heaters/thermometer wiring, and the kinematic mounts. The general goal would be to require less precision of the steps that are executed by hand by choice of components and use of guides. For example, in the kinematic-mounting system, we would like to replace the ball plungers with heavy-duty spring pins with a much larger range of travel. The limited travel of the ball plungers required their careful pre-screening and precise vertical positioning over each filter. 
During the test program, there were several failures ultimately attributed to process and not to design. Tears in an IVCS filter after a vibration test were attributed to loose metal particles found on the carrier. Chipping of the silicon on the edge of a kinematic mount notch after thermal testing was attributed to a pre-existing rough spot on the edge at the point of contact with the ball-end screw, seen in photographs taken before the test. Hairline cracks in the fine mesh in two locations on a DMS filter (and associated tears in the thin film) were attributed to flexing and snapping of the extraction tool used to remove the FC from the ApC-SA after a vibration test. The latter event forced an important redesign of all the FC installation and extraction tools. Note that none of the tears caused a filter to be completely rejected, just lowered in ranking. The DMS tears and cracked mesh noted above were patched using silver epoxy. The IVCS tear was Yshaped and could not be patched, but loss of a single $0.35-\mathrm{mm}$ cell was acceptable according to the thermal and detectornoise budgets. One of the major benefits of the mesh support is that it makes the filters stronger and able to tolerate localized damage.

A programmatic consideration for the use of silicon-mesh FCs is that it integrates an extremely fragile component into a complex sub-assembly that requires an extended test program. As a result, an accident at the sub-assembly stage has considerable cost. To mitigate this cost for SXS, three FC of each size were fabricated and fully tested for flight, which was essentially a choice to pay that cost up front. Additionally, it should be noted that both programmatic risk and mission risk are mitigated by the robustness of the mesh filters, in comparison with the unsupported filters. Although the mesh filters are subject to more handling than the unsupported filters, they are more tolerant of it than the unsupported films would be. And the instrument can tolerate rupture of the film in a few fine-mesh cells, but cannot tolerate loss of an entire filter. Thus the benefit of integrated decontamination heaters and more fault-tolerant filters was determined to be worth the added programmatic cost of integrating and testing a large set of silicon-mesh FCs.

\section{REFERENCES}

[1] Kilbourne, C. A., et al., "The design, implementation, and performance of the ASTRO-H SXS calorimeter array and anti-coincidence detector," Proc. SPIE 9905 (these proceedings) (2016).

[2] Kelley, R.L., et al., "The ASTRO-H high-resolution soft x-ray spectrometer," Proc. SPIE (these proceedings) (2016).

[3] Shirron P. J., et al., "Design and on-orbit operation of the adiabatic demagnetization refrigerator on the ASTRO-H soft X-ray spectrometer instrument," Proc. SPIE 9905 (these proceedings) (2016).

[4] Fujimoto, R., et al., "Performance of the helium dewar and cryocoolers of ASTRO-H SXS," Proc. SPIE 9905 (these proceedings) (2016).

[5] Kelley, R. L., et al., “The Suzaku High Resolution X-Ray Spectrometer,” Publ. Astron. Soc. Japan 59, S77-S112 (2007).

[6] Eckart, M.E., et al., "Ground calibration of the ASTRO-H soft x-ray spectrometer," Proc. SPIE 9905 (these proceedings) (2016).

[7] Speedy, R.J., et al., "The evaporation rate, free energy, and entropy of amorphous water at 150 K," J. Chem. Phys. 105, 240-244 (1996).

[8] Chiao, M. P., et al., "System design and implementation of the detector assembly of the Astro-H soft X-ray spectrometer," Proc. SPIE 9905 (these proceedings) (2016).

[9] Porter, F. S., et al., "In-flight performance of the soft x-ray spectrometer detector system on ASTRO-H," Proc. SPIE 9905 (these proceedings) (2016). 